\title{
Research on Computer Network Security Evaluation Based on Neural Network
}

\author{
Jimin Gao
}

\author{
Department of Computer Engineering, Shenzhen Polytechnic, Shenzhen, China \\ gaojm@szpt.edu.cn
}

Keywords: PSO, Computer network security, Neural network, Evaluation

\begin{abstract}
This paper studies the computer network security problems. There are nonlinear relations among the evaluation indexes, and it is difficult for an accurate mathematical model to describe the nonlinear relationship. In order to improve the evaluation accuracy of computer network security, we put forward a combination model to evaluate the computer network security. The combination model used particle swarm optimization (PSO) to optimize the parameters of BP neural network, speed up the BP neural network's convergence speed, and enhance its global optimization ability, which effectively improved the accuracy of the evaluation model. Simulation results showed that compared with traditional BP neural network model, the combined model, learning ability is faster and global search ability is stronger, which effectively improves the evaluation accuracy of computer network security.
\end{abstract}

\section{Introduction}

The development of network technology has brought convenience to people's life, but also for the virus, Trojans and other destructive programs to provide a convenient network of attack. Computer network security is increasingly dangerous[1]. Accurate and scientific evaluation of the risks faced by the network can reduce the loss caused by computer network security problem[2].

Computer network security is affected by many factors such as intrusion, vulnerability, virus, etc., and a variety of factors are interrelated. There is a complex nonlinear relationship between the factors and the evaluation results. The traditional evaluation methods such as AHP, fault tree analysis and gray model are not only very complicated in computer network security evaluation, but it is difficult to describe the nonlinear relationship accurately, which makes the evaluation precision very low[3]. The expert system is mainly related to the knowledge richness of the experts. The evaluation results are subjective, not objective, and the defects are scientific, so it is not suitable for the complicated computer network security evaluation. In recent years, the rapid development of neural network technology, with self-learning ability, self-organization and strong adaptive ability of artificial intelligence algorithm, the method is simple to achieve and is with strong robustness[4]. Artificial neural network model can effectively overcome the shortcomings of the traditional statistical model, through the adjustment of the connection weights between the neurons, the computer network security and the non-linearity between the various attributes to capture, in order to achieve computer network security accurate evaluation, it is very suitable for computer network security evaluation[5].

\section{The Principle of Computer Network Security Evaluation}

The principle of computer network security evaluation is to determine the content and scope of the evaluation under the guidance of its evaluation standard, analyze the situation of the network, the security situation and the network vulnerability, and then evaluate the relevant evaluation methods[6]. Out of the network security level, the computer network security evaluation mathematical model is as follows:

Network security level $=f\left(x_{1}, x_{2}, \cdots, x_{i}, x_{m}\right)$

In which, $x_{i}$ indicates the computer network security evaluation factor, and $f$ represent the 
computer network security evaluation model

From the computer network security evaluation model, it is very important to select the computer network security evaluation factor and the network evaluation model. Computer network security has the characteristics of uncertainty and non-linearity. Therefore, BP neural network with very strong nonlinear approximation capability is used as the network evaluation model, and the experts choose computer network security factors and score them to determine the weight of the results, so as to improve the accuracy of computer network security evaluation.

\section{The PSO-BPNN Model}

Computer Network Security Evaluation Index System. Based on the management security, physical security and logical security of the computer network system, this paper chooses the computer network safety evaluation index by the experts, as shown in Table 1, and scores the computer network security evaluation index through the expert system to determine its weight.

Table 1 Computer network security evaluation index system

\begin{tabular}{|c|c|c|}
\hline Target layer & Primary index & Secondary index \\
\hline \multirow{12}{*}{$\begin{array}{c}\text { Computer network } \\
\text { security evaluation } \\
\text { index system (A) }\end{array}$} & \multirow{4}{*}{ Manage Security $\left(B_{1}\right)$} & Security Organization System $\left(\mathrm{C}_{11}\right)$ \\
\hline & & Safety management system $\left(\mathrm{C}_{12}\right)$ \\
\hline & & Personnel Safety Training $\left(\mathrm{C}_{13}\right)$ \\
\hline & & Emergency Response Mechanism $\left(\mathrm{C}_{14}\right)$ \\
\hline & \multirow{4}{*}{ Physical security $\left(\mathrm{B}_{2}\right)$} & Network room security $\left(\mathrm{C}_{21}\right)$ \\
\hline & & Power supply safety $\left(\mathrm{C}_{22}\right)$ \\
\hline & & Line Security $\left(\mathrm{C}_{23}\right)$ \\
\hline & & Equipment Safety $\left(\mathrm{C}_{24}\right)$ \\
\hline & \multirow{4}{*}{ Logical security $\left(\mathrm{B}_{3}\right)$} & Data Recovery $\left(\mathrm{C}_{31}\right)$ \\
\hline & & Access Control $\left(\mathrm{C}_{32}\right)$ \\
\hline & & Software signature $\left(\mathrm{C}_{33}\right)$ \\
\hline & & Data encryption $\left(\mathrm{C}_{34}\right)$ \\
\hline
\end{tabular}

Computer Network Security Level Settings. According to the relevant research, the computer network security level is divided into Safety (A), basic safety (B), unsafe (C), extremely unsafe (D). The security level of the total score is set to 1 point, then the corresponding security level and the corresponding score shown in Table 2.

Table 2. Computer network security level

\begin{tabular}{|c|c|c|c|c|}
\hline Level & A & B & C & D \\
\hline Score & $1 \sim 0.85$ & $0.85 \sim 7$ & $0.7 \sim 0.6$ & $0.6 \sim 0$ \\
\hline
\end{tabular}

BP neural Network Algorithm. Neural network is essentially a simulation system for the human brain's thinking process. Its core is the mathematical models and algorithms, and the physical implementation is computer software. Just like thins are composed of atoms, neural network is composed of many interconnected neurons. Fig. 1 shows the basic work principle of neurons, and the mathematical expression is as follows:

$$
y=f\left(\sum w_{i} x_{i}\right)
$$

Where $x_{i}$ and $y$ are respectively the input and output. $w_{i}$ is weight coefficient. $f$ is characteristic function, which reflects the mapping relationship between inputs and outputs, and it is usually a nonlinear function. 


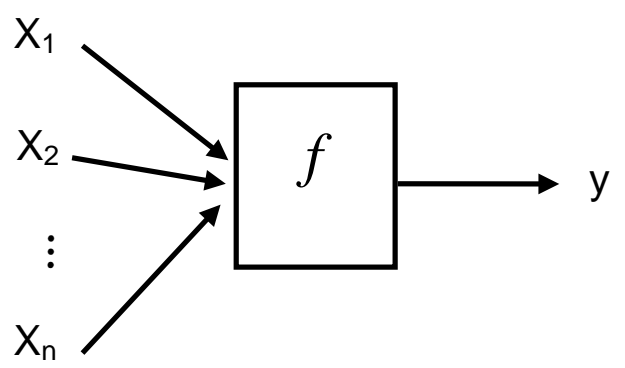

Fig. 1 The neuron model

This seemingly simple model will produce a powerful neural network combing different network topology and network algorithms together. Neural network consists of input layer, hidden layer and output layer, which is shown in Fig. 2.

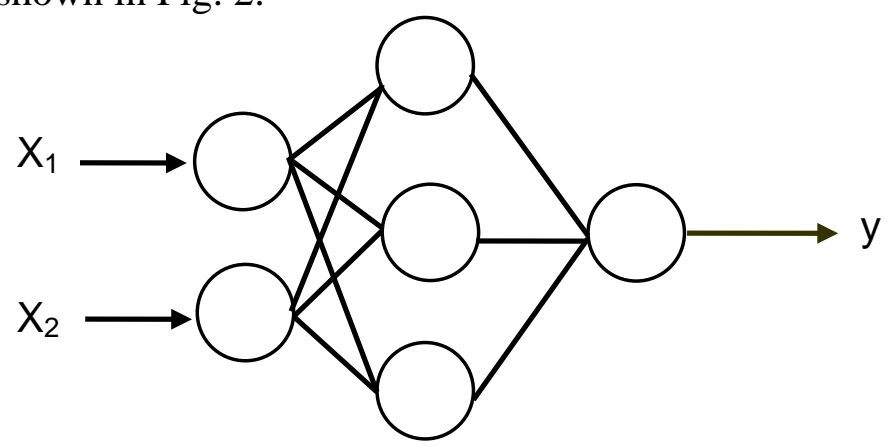

Fig. 2 The connection model of neural network

With a lot of development of neural networks, it was found that no matter how the organizational structure of the network it is, It is always has the following two characteristics:

Self-learning. Neural networks can be modified according to the external environment of their own behavior in order to adapt to the external environment, which is mainly due to its learning process. Learning is often the first step in using neural networks. When a group of information is input, neural networks can continue to adjust its internal parameters (or say weighting coefficient), and eventually produce a series of consistent output.

Generation. Once after the self-learning, the response of neural network, to some extent, to the reducing of input information and their own local defects are no longer sensitive. This mechanism can make the neural network has a strong fault tolerance and reduce the input data quality requirements.

BP neural network has strong nonlinear approximation ability, simple algorithm and easy realization, but it is easy to fall into local extremum, so it is difficult to guarantee convergence to global minimum point, and global search ability is not strong. In addition, the BP neural network is a gradient descent algorithm based on back propagation, and its convergence speed is slow and the learning effect is unsatisfactory. In order to overcome the limitations of the BP neural network itself, this paper uses the PSO algorithm to optimize the BP neural network. The optimization steps are as follows:

(1) Initialize the structure, transfer function, target vector and so on of BP neural network.

(2) Set the size of the particle swarm, the dimension of the parameter, the number of iterations, the momentum coefficient, the initial position of the particle and the initial velocity.

(3) Using the training set to train the BP neural network, the fitness value of each particle is evaluated.

(4) Compare the current value of each particle with the historical best value, and if the current value is better than the historical best value, save the current value of the particle as the historical best value of the individual. Compare the current value of the particle group and Its historical best value, if its current value is better, save the current value for its historical global best value.

(5) Calculate the inertia weight.

(6) The position and velocity of each particle are updated to record the system fitness error for each particle and particle group, respectively. 
(7) Determine the system fitness value error, if the error reaches the set error limit or exceeds the maximum allowable number of iterations, the training is over. The historical global optimal position of the particle is the optimal weight and the optimal threshold of the BP neural network.

Computer Network Security Evaluation Model.

In this paper, the PSO-BPNN model is used to evaluate the computer network security. Firstly, the computer network security evaluation index system is constructed. Then, the BP neural network is optimized by particle swarm optimization algorithm, and the optimal weight and optimal threshold of BP neural network are obtained. Finally, the optimized BP neural network model is used to evaluate the computer network security. The concrete steps are shown in Fig. 3.

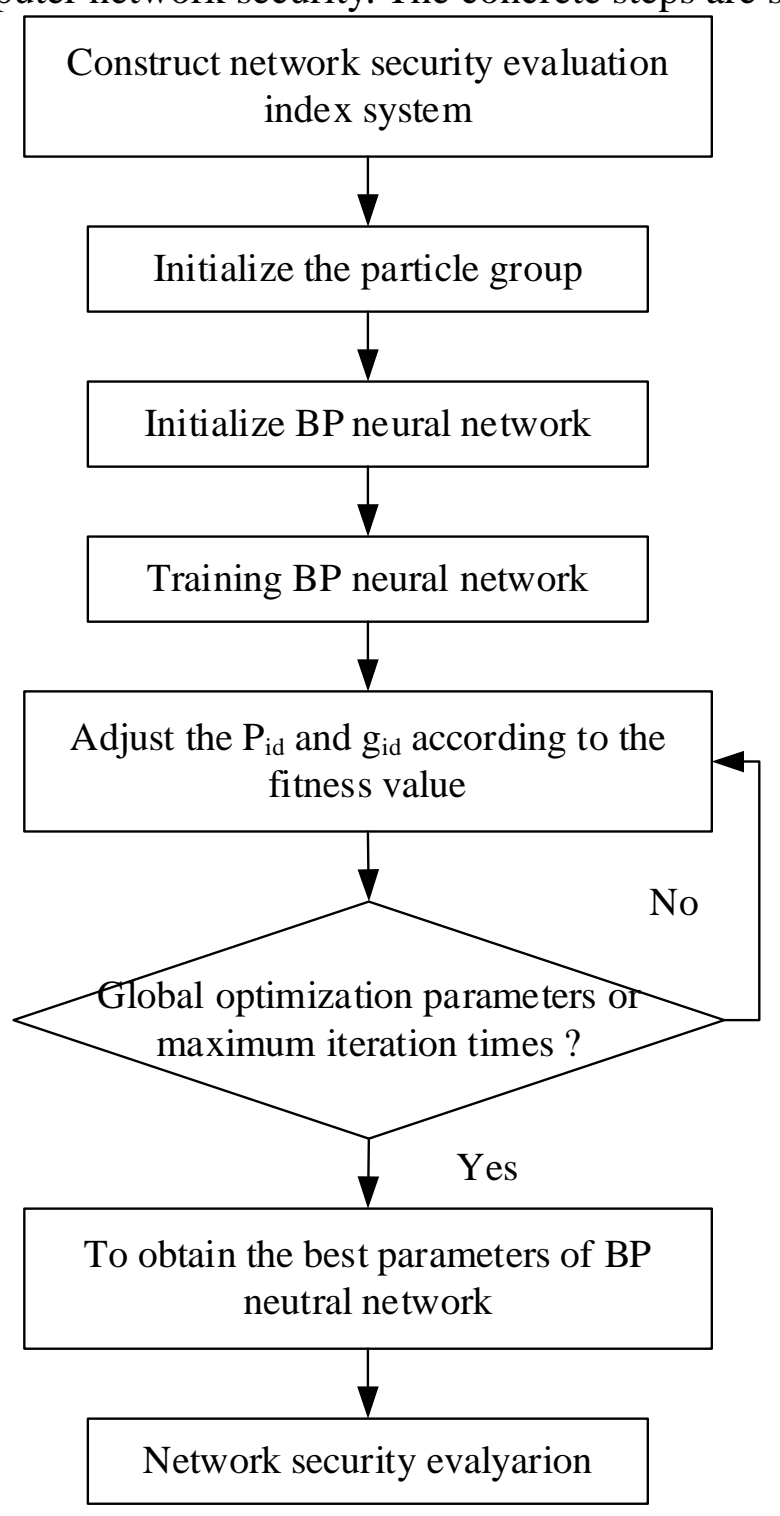

Fig 3. Computer network security evaluation process

\section{Case Analysis}

Experimental Data. Because the data sets related to computer network security can not be shared, this paper collects 50 sets of computer network security evaluation of different sizes of data, and its normalization, as is seen in Table 3. The first 45 sets of data were used as PSO-BPNN training data set, and the last five groups of data were used as test data sets.

Model Parameter Settings. The population size $m=10$, the learning factor $c 1=c 2=2$, all the particles as the BP neural network in all the weights and thresholds, the initial position of the random generation of particles, initialize the particle initial position and velocity, the maximum number of iterations $\mathrm{K}=500$. In order to illustrate the effect of using PSO to optimize the BP neural 
network, this paper uses the traditional BP neural network as the contrast model. The two model selections use traing $\mathrm{dx}$ as the excitation function of the network. The target error is equal to 0.001 .

Results and Analysis. When the two neural networks reached the predetermined learning accuracy, the network was saved and then tested with the test set, the test results were shown in Table 4. Results show that the traditional BP neural network to 47 evaluation results of the computer network security data is wrong, the security level of sample data for class C, which is unsafe, but the evaluation result to highly safety, using PSO to optimize the BP neural network evaluation accuracy reached $100 \%$. According to root mean square error in the two models, the root mean square error of the traditional BP neural network is 0.067 , is much greater than the root mean square error of PSO - BPNN model, its root mean square error is 0.023, which illustrates the use of PSO algorithm, the optimization of BP neural network was successful, not only speed up the convergence speed of the network, and the model to improve the evaluation precision greatly.

Table 3. Computer network security simulation data set

\begin{tabular}{|c|c|c|c|c|c|c|c|c|c|}
\hline No. & 1 & 2 & 3 & 4 & $\cdots$ & 47 & 48 & 49 & 50 \\
\hline C11 & 1 & 1 & 0.4 & 0.8 & $\cdots$ & 0.6 & 0.8 & 0.2 & 0.8 \\
\hline C12 & 0.8 & 1 & 0.4 & 0.8 & $\cdots$ & 0.8 & 0.8 & 0.4 & 0.8 \\
\hline C13 & 0.8 & 0.8 & 0.2 & 0.6 & $\cdots$ & 0.6 & 1 & 0.2 & 0.4 \\
\hline C14 & 0.8 & 0.8 & 0.2 & 0.6 & $\cdots$ & 0.6 & 0.6 & 0.2 & 0.6 \\
\hline C21 & 1 & 1 & 0 & 0 & $\cdots$ & 0 & 1 & 0 & 1 \\
\hline C22 & 0.8 & 0.9 & 0.3 & 0.6 & $\cdots$ & 0.5 & 0.8 & 0.3 & 0.5 \\
\hline C23 & 0.85 & 0.85 & 0.25 & 0.62 & $\cdots$ & 0.65 & 0.8 & 0.3 & 0.6 \\
\hline C24 & 0.8 & 0.8 & 0.4 & 0.6 & $\cdots$ & 0.6 & 0.8 & 0.2 & 0.6 \\
\hline C31 & 0.92 & 0.93 & 0.47 & 0.81 & $\cdots$ & 0.6 & 0.8 & 0.2 & 0.6 \\
\hline C32 & 0.87 & 0.9 & 0.55 & 0.72 & $\cdots$ & 0.75 & 0.85 & 0.37 & 0.77 \\
\hline C33 & 0.85 & 0.9 & 0.45 & 0.75 & $\cdots$ & 0.7 & 0.9 & 0.25 & 0.8 \\
\hline C34 & 0.8 & 1 & 0.4 & 0.6 & $\cdots$ & 0.6 & 0.8 & 0.2 & 0.6 \\
\hline $\begin{array}{c}\text { Expected } \\
\text { output }\end{array}$ & 0.85 & 0.88 & 0.33 & 0.67 & $\cdots$ & 0.61 & 0.85 & 0.24 & 0.63 \\
\hline
\end{tabular}

Table 4. Comparison of evaluation results

\begin{tabular}{|c|c|c|c|c|}
\hline Samples & Security Level & Expected output & BPNN & PSO-BPNN \\
\hline 46 & $\mathrm{D}$ & 0.46 & 0.41 & 0.44 \\
\hline 47 & $\mathrm{C}$ & 0.61 & 0.55 & 0.64 \\
\hline 48 & A & 0.85 & 0.76 & 0.86 \\
\hline 49 & $\mathrm{D}$ & 0.24 & 0.19 & 0.26 \\
\hline 50 & $\mathrm{C}$ & 0.63 & 0.69 & 0.66 \\
\hline \multicolumn{3}{|c|}{ Root mean square error } & 0.067 & 0.023 \\
\hline
\end{tabular}

\section{Conclusions}

In this paper, the PSO algorithm is used to optimize the BP neural network. The BP neural network is used to solve the problem of computer network security. The simulation results show that the global search ability, evaluation accuracy and convergence rate of the BP neural network model optimized by PSO algorithm are significantly higher than that of the traditional BP neural network, and the optimization of BP neural network by PSO algorithm is successful. Our network security evaluation model provides a new guidance for the related research in the future. 


\section{References}

[1] Luo B, Liu Y. The risk evaluation model of network information security based on improved BP neural network[C]// International Symposium on Instrumentation \& Measurement, Sensor Network and Automation. IEEE, 2012:189-191.

[2] Zhang Y B, Yan Z Q. Researches on the Network Security Evaluation Method Based on BP Neural Network[J]. Applied Mechanics \& Materials, 2014, 686:470-473.

[3] Zhang Y L, Xiong J L. Research of computer network security evaluation based on RBF neural network[C]// International Conference on Machinery, Materials and Computing Technology. 2016.

[4] Jin S. Research on Application of Neural Network in Computer Network Security Evaluation[J]. Open Electrical \& Electronic Engineering Journal, 2014, 8(1):766-771.

[5] Zhan J, University J. Evaluation of computer network security based on adaptive BP neural network[J]. Modern Electronics Technique, 2015.

[6] Ren-Jie WU. Application Research of Computer Network Security Evaluation Based on Network Security[J]. Computer Simulation, 2011, 28(11):126-129. 